\title{
ON A THEOREM OF ISEKJ
}

\author{
by PETER KESSLER
}

(Received 4 March, 1969)

1. The purpose of this paper is to generalize a result of $\mathrm{K}$. Iseki [1]. In his note, Iseki proves that, in a normal space $S$, for every countable discrete collection $\mathscr{H}=\left\{H_{1}, H_{2}, \ldots\right\}$ of sets from $S$, there exists a countable collection $\mathscr{U}=\left\{U_{1}, U_{2}, \ldots\right\}$ of mutually disjoint open sets from $S$ such that $\bar{H}_{i} \subset U_{i}$ for every $i$.

In this paper we consider almost discrete, separated and completely separated collections of sets from a topological space. It is shown that the analogous property holds for almost discrete collections in a normal space, and for separated collections in a completely normal space. The well-known property that a topological space is normal if and only if any two closed disjoint sets are completely separated, is here expressed in terms of completely separated discrete collections.

2. Definitions. Let $S$ be a topological space. We denote by $\mathscr{H}=\left\{H_{\alpha}: \alpha \in \Omega\right\}$ an arbitrary collection of sets from $S$. For a collection $\mathscr{H}$, we denote by $\overline{\mathscr{H}}$ the collection of closures of sets from $\mathscr{H}$. Open collections, i.e. collections of open sets, will be denoted by $\mathscr{U}, \mathscr{V}$.

A collection $\mathscr{H}=\left\{H_{\alpha}: \alpha \in \Omega\right\}$ is called discrete if it satisfies the following two conditions:

(1) $\bar{H}_{\alpha} \cap \bar{H}_{\beta}=\emptyset$ for every $\alpha, \beta \in \Omega, \alpha \neq \beta$;

(2) for an arbitrary subset $\Gamma$ of $\Omega, \bigcup_{\alpha \in \Gamma} \bar{H}_{\alpha}=\overline{\bigcup_{\alpha \in \Gamma} H_{\alpha}}$.

If $\mathscr{H}$ is discrete, then $\overline{\mathscr{H}}$ is also discrete. For every discrete collection $\mathscr{H}, \bigcup_{\alpha \in \Omega} \bar{H}_{\alpha}$ is a closed set in $S$.

From the above definition, with some modifications, we get the following definition for almost discrete collections:

A collection $\mathscr{H}=\left\{H_{\alpha}: \alpha \in \Omega\right\}$ is almost discrete if, for every $\alpha \in \Omega$,

$$
\bar{H}_{\alpha} \cap \overline{\substack{\beta \in \Omega \\ \beta \neq \alpha}} \bar{\bigcup}_{\beta}=\emptyset .
$$

It follows that, for such a collection, for every $\alpha^{\prime}, \alpha^{\prime \prime} \in \Omega$ with $\alpha^{\prime} \neq \alpha^{\prime \prime}$, we have $\vec{H}_{\alpha^{\prime}} \cap \bar{H}_{\alpha^{\prime \prime}}=$ $\emptyset$. Every discrete collection is almost discrete, but it is very easy to verify that the converse is not true. From the definition it follows that, if $\mathscr{H}$ is almost discrete, then $\overline{\mathscr{H}}$ is almost discrete.

A collection $\mathscr{H}=\left\{H_{\alpha}: \alpha \in \Omega\right\}$ is separated if, for every $\alpha \in \Omega$,

$$
H_{\alpha} \cap \overline{\substack{\beta \in \Omega \\ \beta \neq \alpha}} H_{\beta}=\emptyset .
$$

Every almost discrete collection is separated, but the converse is not true. Every collection of mutually disjoint open sets is separated. If $\mathscr{H}=\left\{H_{\alpha}: \alpha \in \Omega\right\}$ is separated, then, for every $\alpha \in \Omega$, we have $\bar{H}_{\alpha} \cap \underset{\substack{\beta \in \Omega \\ \beta \neq \alpha}}{\bigcup} H_{\beta}=\emptyset$, i.e. the sets $H_{\alpha}$ and $\bigcup_{\substack{\beta \in \Omega \\ \beta \neq \alpha}} H_{\beta}$ are separated in the usual sense for 
every $\alpha \in \Omega$. The condition $\bar{H}_{\alpha} \cap \bigcup_{\substack{\beta \in \Omega \\ \beta \neq \alpha}} H_{\beta}=\emptyset$ for every $\alpha \in \Omega$, does not imply that the collection is separated.

For countable collections we introduce the concept of completely separated collection in the following way:

A countable collection $\mathscr{H}=\left\{H_{1}, H_{2}, \ldots\right\}$ is completely separated if, for every sequence $a_{1}, a_{2}, \ldots$ of real numbers, there exists a continuous function $f$ on $S$ such that $f\left(H_{i}\right)=a_{i}$ for every $i$.

Two completely separated sets in the usual sense form a completely separated collection. Every countable completely separated collection is discrete.

3. Normal spaces. In a normal space, the following additional properties hold for almost discrete collections:

Lemma. 1. Let $S$ be a normal space. If, for every almost discrete collection $\mathscr{H}=$ $\left\{H_{\alpha}: \alpha \in \Omega\right\}$, there exists a collection $\mathscr{V}=\left\{V_{\alpha}: \alpha \in \Omega\right\}$ of mutually disjoint open sets such that, for every $\alpha \in \dot{\Omega}$, we have $\vec{H}_{\alpha} \subset V_{\alpha}$, then there exists an almost discrete collection $\mathscr{U}=\left\{U_{\alpha}: \alpha \in \Omega\right\}$ of open sets such that $\bar{H}_{\alpha} \subset U_{\alpha}$ for every $\alpha \in \Omega$.

Proof. For every $\alpha \in \Omega$, let $U_{\alpha}$ be an open set such that $\bar{H}_{\alpha} \subset U_{\alpha} \subset \bar{U}_{\alpha} \subset V_{\alpha}$; then

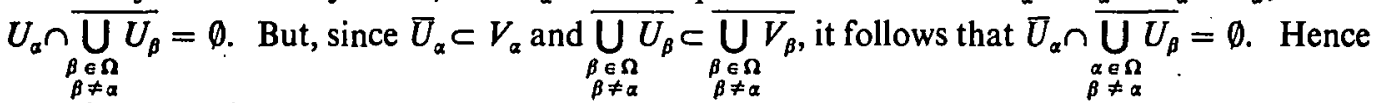
$\mathscr{U}=\left\{U_{\alpha}: \alpha \in \Omega\right\}$ is almost discrete.

THEOREM 1. A topological space $S$ is normal if and only if, for every countable almost discrete collection $\mathscr{H}=\left\{H_{1}, H_{2}, \ldots\right\}$, there exists an almost discrete collection $\mathscr{V}=\left\{V_{1}, V_{2}, \ldots\right\}$ of open sets such that $\bar{H}_{i} \subset V_{i}$ for every $i$.

Proof. The condition of the theorem is evidently sufficient for normality of $S$.

For necessity, let $S$ be a normal space and let $\mathscr{H}=\left\{H_{1}, H_{2}, \ldots\right\}$ be a countable almost discrete collection of sets from $S$. We prove the following assertion $P(n)$ :

$P(n)$ : There exist open sets $V_{1}, V_{2}, \ldots, V_{n}, V^{n+1}$ such that

(a) $\bar{H}_{i} \subset V_{i}$ for $i=1,2, \ldots, n$,
(b) $\bigcup_{k=n+1}^{\infty} H_{k} \subset V^{n+1}$
(c) $V_{k} \cap V_{i}=\emptyset$ for $i<k \leqq n$ and
(d) $\bigcup_{i=1}^{n} V_{i} \cap V^{n+1}=\emptyset$. 
For $n=1$, the sets $\bar{H}_{1}$ and $\overline{\bigcup_{k=2}^{\infty} H_{k}}$ are closed and disjoint in $S$. Hence there exist open sets $V_{1}$ and $V^{2}$ such that $(a) \bar{H}_{1} \subset V_{1},(b) \bigcup_{k=2}^{\infty} H_{k} \subset V^{2}$ and $(d) V_{1} \cap V^{2}=\emptyset$.

We assume that $P(n)$ is valid for $n$. There exists an open set $U^{n+1}$ such that

$$
\overline{\bigcup_{k=n+1}^{\infty} H_{k}} \subset U^{n+1} \subset \overline{U^{n+1}} \subset V^{n+1} .
$$

Then $\overline{U^{n+1}}$ is a normal subspace of $S$ and $\mathscr{H}_{n+1}=\left\{H_{n+1}, H_{n+2}, \ldots\right\}$ is almost discrete in $\overline{U^{n+1}}$. There exist the sets $V_{1}^{n+1}$ and $V_{2}^{n+1}$, open in $\overline{U^{n+1}}$, such that $\overline{H_{n+1}} \subset V_{1}^{n+1}, \overline{\bigcup_{k=n+2}^{\infty} H_{k}} \subset$ $V_{2}^{n+1}$ and $V_{1}^{n+1} \cap V_{2}^{n+1}=\emptyset$. Then there exist $W_{1}^{n+1}$ and $W_{2}^{n+1}$, open in $S$, such that $V_{1}^{n+1}=$ $\overline{U^{n+1}} \cap W_{1}^{n+1}$ and $V_{2}^{n+1}=\overline{U^{n+1}} \cap W_{2}^{n+1}$. Now let $V_{n+1}=U^{n+1} \cap W_{1}^{n+1}, V^{n+2}=U^{n+1} \cap W_{2}^{n+1}$. The sets $V_{1}, V_{2}, \ldots, V_{n+1}, V^{n+2}$ satisfy $P(n+1)$.

In this way we construct a sequence of open sets $V_{1}, V_{2}, \ldots$ with the required properties, i.e. $F_{i} \subset V_{i}(i=1,2, \ldots)$ and $V_{i} \cap V_{j}=\emptyset(i \neq j)$. The theorem follows then from Lemma 1.

As a consequence we obtain Iseki's theorem:

THeOREM 2 (Iseki). A topological space $S$ is normal if and only if, for every countable discrete collection $\mathscr{H}=\left\{H_{1}, H_{2}, \ldots\right\}$, there exists a countable discrete collection of open sets $\mathscr{U}=\left\{U_{1}, U_{2}, \ldots\right\}$ such that $\bar{H}_{i} \subset U_{i}$ for every $i$.

Now let $\xi$ be the collection of countable collections of mutually disjoint sets from a topological space $S$ which satisfies the following conditions:

(a) If $\mathscr{H} \in \xi$, then $\overline{\mathscr{H}} \in \xi$,

(b) for every $\mathscr{H}=\left\{H_{1}, H_{2}, \ldots\right\}$ from $\xi$, there exists a collection of open sets $\mathscr{U}=$ $\left\{U_{1}, U_{2}, \ldots\right\}$ from $\xi$ such that $H_{i} \subset U_{i}$ for every $i$.

Such a collection $\xi$ contains, as it is easy to verify, only almost discrete collections. The following theorem holds:

THEOREM 3. A topological space $S$ is normal if and only if $\xi$ consists exactly of all countable almost discrete collections.

For completely separated collections the following theorem holds:

THEOREM 4. A topological space $S$ is normal if and only if every countable discrete collection of sets from $S$ is completely separated.

Proof. For collections of two sets, this is Urysohn's lemma. Therefore it follows immediately that the condition of the theorem is sufficient for normality of $S$.

For necessity, let $S$ be normal, and let $\mathscr{H}=\left\{H_{1}, H_{2}, \ldots\right\}$ be a discrete collection in $S$. There exists a discrete collection $\mathscr{U}=\left\{U_{1}, U_{2}, \ldots\right\}$ of open sets such that $\bar{H}_{i} \subset U_{i}$. Let $a_{1}, a_{2}, \ldots$ be an arbitrary sequence of real numbers. For every pair $\left(H_{i}, U_{i}\right)$, there exists a continuous function $f_{i}$ such that $f_{i}\left(H_{i}\right)=a_{i}$ and $f_{i}\left(S-U_{i}\right)=0$. We consider then $f=\sum_{i=1}^{\infty} f$. Evidently $f\left(H_{i}\right)=a_{i}$. It is easy to verify that the function $f$ is continuous. 
4. Completely normal spaces. A similar characterization with separated collections is possible for complete normality.

THEOREM 5. A topological space $S$ is completely normal if and only if, for every countable separated collection $\mathscr{H}=\left\{H_{1}, H_{2}, \ldots\right\}$, there exists a countable separated collection $\mathscr{U}=\left\{U_{1}, U_{2}, \ldots\right\}$ of open sets such that $H_{i} \subset U_{i}$ for every $i$.

The proof of Theorem 5 is analogous to the proof of Theorem 1, and is here omitted.

Let $\xi^{\prime}$ be the collection of all countable collections of mutually disjoint sets from a topological space $S$ which satisfies the condition:

If $\mathscr{H}=\left\{H_{1}, H_{2}, \ldots\right\}$ is from $\xi^{\prime}$, then there exists a collection $\mathscr{U}=\left\{U_{1}, U_{2}, \ldots\right\}$ of open sets from $\xi^{\prime}$ such that $H_{i} \subset U_{i}$ for every $i$.

Every collection from $\xi^{\prime}$ is separated, and the following theorem holds:

TheоRem 6. A topological space $S$ is completely normal if and only if $\xi^{\prime}$ consists exactly of all countable separated collections from $S$.

\section{REFERENCES}

1. K. Iseki, A note on normal spaces, Math. Japon. 3 (1953), 45.

\section{UNIVERSITY OF CRAIOVA}

Craiova, Roumania 